\title{
Percepciones de la comunidad educativa sobre estudiantes migrantes en Chile: trato, diferencias e inclusión escolar*
}

\author{
Perceptions of the educational community about migrant students in Chile: \\ treatment, differences and school inclusion
}

\author{
Iskra Pavez-Soto ${ }^{a}$,Juan Ortiz-López ${ }^{b}$, Anastassia Domaica-Barrales ${ }^{a}$ \\ ${ }^{a}$ Universidad Bernardo O’Higgins, Centro de Investigación en Educación (CIE). \\ iskra.pavez@ubo.cl, anastassia.domaica@gmail.com \\ ${ }^{b}$ Universidad de Playa Ancha, Instituto Interuniversitario de Investigación Educativa (IESED Chile). \\ juaneduardoortiz@gmail.com
}

\section{RESUMEN}

La posibilidad de promover formas activas de inclusión en centros educativos con presencia de estudiantes extranjeros queda determinada, muchas veces, por las percepciones que tiene el cuerpo docente sobre el sujeto migrante. En ese marco, el objetivo de este artículo es analizar las percepciones que tienen las comunidades educativas de las regiones con mayor presencia de población migrante en relación al trato, las diferencias y la forma en que se aborda la inclusión de las y los estudiantes extranjeros. Mediante la aplicación de entrevistas semiestructuradas a diversos actores se pudo establecer que el buen trato y la inclusión en el contexto escolar depende de los niveles de asimilación de las y los estudiantes migrantes, quienes además son racializados y sexualizados, especialmente las niñas afrocolombianas. En forma adicional, se observa un discurso nacionalista que tiende a la estigmatización de la infancia migrante. Por lo tanto, se requieren políticas públicas de inclusión escolar.

Palabras claves: educación, migración, buen trato, racialización, sexualización.

\section{ABSTRACT}

The possibility of promoting active forms of inclusion in educational centers with the presence of foreign students is often determined by the perceptions that exist around the migrant subject. In this framework, the objective of this article is to analyze the perceptions that educational communities of the regions with the greatest presence of migrant population have in relation to treatment, differences and the way in which inclusion of foreign students is addressed. Through the application of semi-structured interviews to different actors, it was established that good treatment and inclusion in the school context depend on the assimilation levels of migrant students, who are also racialized and sexualized, especially Afro-Colombian girls. Additionally, there is a nationalist discourse that tends to stigmatize migrant children. Therefore, public policies for school inclusion are needed.

Key words: education, migration, good treatment, racialization, sexualization.

* En este artículo se presentan resultados preliminares del Proyecto FONDECYT Regular No 1170947 "Múltiples violencias que afectan a las niñas y los niños migrantes en Chile. Derechos, intervenciones sociales y políticas públicas", dirigido por Dra. Iskra Pavez-Soto. Agradecemos al programa FONDECYT (Fondo Nacional de Desarrollo Científico y Tecnológico) de CONICYT (Comisión Nacional de Ciencia y Tecnología) del Gobierno de Chile, por haber financiado este estudio. 
Estudios Pedagógicos XLV, N 3: 163-183, 2019

PERCEPCIONES DE LA COMUNIDAD EDUCATIVA SOBRE ESTUDIANTES MIGRANTES EN CHILE: TRATO, DIFERENCIAS E INCLUSIÓN ESCOLAR

\section{INTRODUCCIÓN}

El objetivo de este artículo es analizar diferentes percepciones que tienen las y los profesionales de la comunidad educativa, como docentes, paradocentes, psicólogos y directivos, con relación al trato, diferencias y la inclusión al interior de instituciones educacionales de acogida de estudiantes migrantes en tres regiones de Chile con mayor presencia de población extranjera: Antofagasta, Valparaíso y Región Metropolitana (Departamento de Extranjería y Migración, 2017). Estos resultados forman parte de un estudio FONDECYT (ANONIMIZADO) que indaga sobre las percepciones del cuerpo docente vinculadas al trato y las diferencias en los procesos de inclusión escolar de estudiantes migrantes en Chile.

De acuerdo a los últimos datos entregados por el Instituto Nacional de Estadísticas y el Departamento de Extranjería (en adelante INE-DEM, 2019) en Chile residen 1.251.225 personas extranjeras, de las cuales 646.128 son hombres y 605.097 son mujeres, quienes provienen de países como Venezuela (con 288.233 personas, que representan el $23 \%$ del total de población migrante), Perú (con 223.923, 18\%), Haití (179.338, 14,3\%), Colombia (146.582, 12\%), Bolivia (107.346, 9\%), Argentina (74.713, 6\%) y Ecuador $(36.994,3 \%)$. Dentro de esta población, se estima que 183.315 serían niñas, niños y adolescentes entre 0 y 19 años de edad, lo que correspondería al 14,7\% (INE-DEM, 2019).

El número de niñas y niños migrantes en el sistema escolar chileno sería de 77.608 , lo que correspondería a un 2,2\% del total (Subsecretaría de la Educación, 2017). Si bien la cifra es baja, su presencia ha ido aumentando. En este escenario, la inclusión es una tarea ardua y constante de la comunidad educativa, especialmente para las y los profesionales de la educación, porque aún operan obstáculos y oportunidades, así como también existen distintas concepciones de los derechos, el trato justo, el currículum y la puesta en marcha de dinámicas acordes a las nuevas exigencias del sistema escolar.

La llegada de población migrante en edad escolar a Chile ha generado un conjunto de desafíos para el Estado y el sistema educativo, pero especialmente ha sido una oportunidad de revisión, aprendizajes y cambios en las escuelas donde reciben a niñas y niños extranjeros, instalando la preocupación por alcanzar la inclusión y el respeto de sus derechos (Cerón, Pérez y Poblete, 2017). En efecto, la presencia de población migrante en las aulas habitualmente plantea nuevas inquietudes al sistema educacional en todos sus niveles, abriendo un debate que implica posiciones político-pedagógicas, expectativas educativas y también socioculturales (Jiménez y Fardella, 2015).

Si bien, durante los últimos años, el estado chileno ha impulsado una serie de dispositivos legislativos y educacionales cuya finalidad es garantizar una educación inclusiva, en cuanto al estudiantado migrante, todavía es insuficiente (Belmar et al., 2019). Con la llegada de población migrante, el sistema educativo debería transitar desde una lógica de competencia y homogeneización hacia una lógica de inclusión, respeto por la diferencia y participación; estas transformaciones permitirían construir sociedades más cohesionadas, inclusivas y pacíficas y, con esto, lograr un mayor bienestar individual y social.

En este sentido, es necesario considerar de forma prioritaria las cuatro dimensiones de los derechos de la infancia en el contexto educativo: no discriminación, identidad, participación y buen trato. Además, se debe atender a los procesos de inclusión educativa, entre ellos; el derecho al acceso, permanencia, promoción, participación y calidad de la educación, sin infligir discriminación alguna por factores como sexo, identidad sexual, 
credo, origen étnico, entre otros. Es labor del Estado resguardar el cumplimiento de estos derechos, de modo que cada vez que no se cumpla o se vea amenazado, las instituciones sociales y la sociedad civil se pronuncien bajo el irrestricto reconocimiento de la dignidad de las personas (Superintendencia de Educación, 2017). En el contexto educativo y en la realidad que experimentan las y los profesionales de la educación, lo anterior resulta complejo, considerando la escasa formación inicial en estas áreas (Peña-Sandoval, 2017; Ortiz-López, 2017), unido al urgente desafío de actuar en comunidades escolares cada día más diversas, debido al ascenso del fenómeno migratorio en Chile. Existen varios factores que alteran la convivencia escolar, la relación de estudiantes, profesores y la comunidad educativa en todas sus dimensiones como, por ejemplo, las barreras idiomáticas con estudiantes haitianos (Pavez-Soto et al., 2018). La Agencia de Calidad de la Educación (2017) indica la preocupación sobre el trato que reciben las y los estudiantes (en general, no solo migrantes) en la sala de clases, pues el maltrato y la violencia, por cierto, guardan relación con conductas normalizadas como no saludar, evitar, excluir, discriminar, mentir, descalificar, criticar destructivamente, difundir rumores, entre otros; cuyos efectos no se han evaluado a la fecha. Según los datos del Ministerio de Educación (MINEDUC, 2011), un $44,7 \%$ de las y los estudiantes en Chile declara haber sido agredido en el colegio y más de la mitad señala que el cuerpo directivo y docente no percibe las situaciones de acoso escolar. Sin duda, son cifras que debieran preocuparnos.

\section{MARCO TEÓRICO}

La presencia de población migrante en edad escolar genera un conjunto de desafíos a los sistemas educacionales en los que se insertan (Poblete, 2018). Porque, no se trata solo de elementos asociados al acceso, sino también en cuanto a la formación ciudadana en un mundo globalizado (Johnson, 2015), asumiendo la condición plural y multicultural de las sociedades actuales, en especial de aquellas receptoras de migración. En ese marco, el reconocimiento y visibilidad de la diversidad aparece como un valor relevante de los sistemas educacionales, al ser el primer paso para avanzar hacia formas activas de inclusión educativa. En tal sentido, la inclusión alude a diversos elementos, entre los que destacan aspectos éticos vinculados a la perspectiva de derechos humanos (Echeita, 2008), en la que se asume que todas y todos pueden ejercer el derecho a la educación, en cuanto al acceso y participación en los procesos educativos, "al margen de cualquier consideración relacionada con la etnia, género, estilos de aprendizaje o necesidades educativas especiales" (Poblete y Galaz, 2017, p. 42).

En rigor, la educación inclusiva debiese ser capaz de cuestionar la matriz sociohistórica de la dominación (Ocampo, 2016), superando las exclusiones de los distintos grupos que entran en articulación en los sistemas educativos, en especial de quienes han sido marginalizados.

Si bien la inclusión remite a una aspiración y un principio ético, no se puede desconocer que requiere de un fuerte componente procedimental que implica una revisión de las políticas, las prácticas y las culturas educativas, para identificar y eliminar las barreras que impiden la participación equitativa en el sistema educacional (Booth y Ainscow, 2000). Lo anterior plantea cambios y transformaciones sustantivas en las formas de trabajo docente, elementos curriculares, análisis de políticas y formas de convivencia que involucran a toda la comunidad educativa. 
De ahí que el rol de "una escuela plural y democrática es abrirse institucional, organizativa y curricularmente hacia las diversas formas culturales y de socialización que caracterizan a sus estudiantes, docentes y el entorno donde se ubica" (Poblete, 2009, p. 186). Por lo mismo, es clave la manera en que las comunidades educativas perciben y valoran la diversidad, en particular aquellas que encarnan la población migrante.

Para el caso de las sociedades latinoamericanas esto adquiere mayor importancia, dado que como afirma Novaro (2016) ha existido una intención clara en el accionar del Estado y sus instituciones por generar una identidad uniforme, para lo cual la escuela ha sido el principal instrumento asimilador. En esa misma línea, diversos análisis sitúan a Chile como un país que se ha concebido a sí mismo como una isla (Johnson, 2015), en el que la escuela se ha "ordenado desde una perspectiva normalizadora y excluyente, que apuesta por la construcción de una identidad uniforma y homogénea" (Poblete, 2018, p. 52).

Por cierto, los procesos migratorios han tensionado la construcción de esta identidad nacional, afectando las percepciones que se tienen en torno al sujeto migrante. De hecho, el aumento sostenido de estudiantes extranjeros ha comenzado a ser percibido como una amenaza (Ortiz, 2008) y como uno de los principales problemas que enfrentan los sistemas educativos actuales. Las formas de reaccionar frente a la presencia migrante ha sido mediante prácticas asimilacionistas, las que finalmente "inciden en el contexto escolar, en las relaciones y los procesos de enseñanza aprendizaje que tienen lugar en él" (Ortiz, 2008, p. 254).

Las evidencias muestran que suele atribuirse un déficit a las y los estudiantes migrantes, que se expresa en rendimientos descendidos, dificultad para adaptarse a las normas y prejuicios que están presentes en las comunidades educativas, en especial, en las y los docentes (Cerón et al., 2017). En efecto, en este imaginario niñas y niños migrantes no alcanzan las competencias requeridas para recibir una enseñanza efectiva o participar en los códigos que posee el sistema escolar (Kaluf, 2009).

En ese marco, el paso por el sistema educacional, concebido como un dispositivo de poder y control (Foucault, 1977; Bernstein, 1990; Bourdieu y Passeron, 2001), configura trayectorias diferenciadas que son resentidas en mayor medida por quienes pertenecen a grupos minoritarios (Gibson, 1998; Ogbu, 1991; Giroux, 1994), ya que la escuela está pensada para quienes pertenecen a la cultura mayoritaria. De esta forma, las percepciones y acciones en torno a las y los estudiantes extranjeros por parte de la comunidad educativa, tiene una incidencia directa en las posibilidades de inclusión que se puedan generar a nivel de escuelas y liceos. Mientras que, si esta percepción es positiva, mayores serán sus posibilidades de acceso y participación equitativa en los aprendizajes y el conjunto de acciones propias del espacio escolar. Sin embargo, existe evidencia que el cuerpo docente tiene imaginarios negativos respecto al estudiantado migrante, vinculados al déficit académico y un comportamiento negativo, generando etiquetas en torno a algunos colectivos e incluso normalizando prácticas discriminatorias, lo que finalmente ha cristalizado en una "concepción de la diversidad que representan los y las migrantes como una suerte de obstáculo que debe ser superado" (Cerón et al., 2017, p. 242).

Diversos autores (Jordán, 1994; Poblete, 2009; León, Mira y Gómez, 2007) sostienen que más relevante que las competencias o conocimientos que puedan tener y desplegar las y los docentes en los espacios educativos, son las actitudes hacia la diversidad y las percepciones que puedan expresar en torno a ésta, porque de esto dependerá la forma en que configuran las relaciones con las y los estudiantes, su rendimiento y especialmente la posibilidad de aportar a una cultura inclusiva al interior de escuelas o liceos (Cerón et 
al., 2017). Precisamente, una cultura inclusiva involucra a toda la comunidad educativa, comprometiéndose en la promoción de valores democráticos y solidarios, basados en el respeto irrestricto de la diversidad (Blanco, 2005; Booth y Aisncow, 2000; Echeita, 2008). Sin embargo, los propios estudiantes nacionales también expresan niveles de prejuicio preocupantes hacia la población extranjera, generando incluso categorías jerárquicas en torno a ellos (Unicef, 2004; 2011), por lo que su experiencia educativa no siempre se condice con los principios de la inclusión, por el contrario, "está fuertemente marcada por las constantes situaciones de discriminación y racismo de las cuales son víctimas" (PavezSoto, 2012, p. 87). Al respecto, "el color de la piel, la forma de hablar y otras características distintivas se constituyen en elementos que generan marginación por parte de niños y niñas chilenas" (Poblete y Galaz, 2017, p. 241).

De esta forma, existe un escenario complejo en torno al tránsito de las y los estudiantes migrantes por el sistema educacional, esto, porque las formas de aprovechamiento escolar de los grupos minoritarios en los sistemas educacionales son fenómenos variables (Ogbu, 1991; McCarthy, 1994), que dependen también de los estilos de acogida que existen previamente en escuelas y liceos. De ahí, entonces, el valor de reconocer en la percepción de las comunidades educativas hacia la población migrante, una variable relevante de estudio.

\section{METODOLOGÍA}

La metodología de la investigación ha sido cualitativa, pues nos permite construir y develar los significados que emanan de las experiencias de los sujetos a través de sus discursos. Además, el diseño de la investigación comprende la recopilación de información desde un enfoque de estudio multicaso (Stake, 2010), en tanto cada sujeto pertenece a una realidad distinta (región y contexto escolar), sin embargo, aborda y explora el mismo fenómeno investigado.

La recolección de datos se realizó en las regiones de Antofagasta, Valparaíso y Santiago de Chile, desde abril a diciembre del año 2017, correspondiendo a un diseño transversal, porque se abordó en un tiempo acotado (Vogt, Gardner \& Haeffele, 2012). La técnica de recolección de datos utilizada fue la entrevista semiestructurada, pues permite la flexibilización de los protocolos en base a las respuestas de los sujetos y explora las experiencias vividas como narraciones complejas que necesitan de contextualización y de relación teórica. Los datos se trataron mediante la técnica de análisis del discurso, que comprende al texto como macrounidad de análisis, en donde es posible observar la representación del fenómeno investigado (Krippendorff, 2018), al tiempo que permite indagar sobre los constructos sociales y los significados que a ellos se adhieren a través del discurso (Dunn \& Neumann, 2016).

La codificación de los textos obtenidos se realizó de manera inductiva y deductiva, es decir, se elaboraron categorías analíticas a partir de los textos, pero también se establecieron categorías a priori. En cuanto al proceso de codificación, se llevó a cabo una primera pre-codificación en búsqueda de las categorías generadas deductivamente y para sondear aquellas que surgieran desde los discursos. Posteriormente, se codificó de manera sustantiva o 'en vivo', pues contemplan “conocer mejor los significados específicos de los participantes y sus experiencias en relación con estos significados" (Monge, 2015, p. 80). 
Este proceso de codificación se realizó utilizando el software MAXQDA® versión 12, para efectos tanto de codificación como de creación de mapas semánticos que permitieran analizar las relaciones entre los códigos, categorías analíticas y las coocurrencias entre los mismos. Los mapas semánticos nos permitieron identificar las relaciones discursivas a nivel conceptual e inferencial, por lo que cada categoría desarrollada se basa en la generación de relaciones textuales e intertextuales. Dichos mapas ratificaron la estructura de significados y la relación entre ellos respecto del fenómeno estudiado.

La muestra del estudio fue de tipo opinático-estratégico (Valles, 2003), porque el equipo de investigación determinó una serie de criterios de selección, hasta que se saturó la información. La muestra estuvo compuesta por 16 docentes, directivos y profesionales llamados asistentes de la educación que atienden a niñas y niños migrantes en su trabajo cotidiano.

En cuanto a los aspectos éticos, se estableció un protocolo a través de la firma de Formularios de Consentimiento Informado para garantizar el acceso a la información durante el proceso investigativo, sus derechos como participantes a abandonar el estudio cuando lo requiriesen, los beneficios y riesgos de participar en el estudio, como también la seguridad en cuanto al almacenamiento de los datos y la protección de las identidades de las y los participantes, lo que incluye el anonimato en la diseminación de resultados a través del uso de pseudónimos.

\section{RESULTADOS}

A continuación, presentamos los resultados del análisis de los discursos, lo que resultó en tres categorías principales, las que fueron obtenidas deductivamente: inclusión escolar, diferencias y comparaciones, y trato. Cada una de estas categorías derivaron en otras subcategorías analíticas, las que fueron obtenidas de manera emergente desde el texto. La codificación realizada a los textos originó distintos mapas semánticos por categoría, los que presentaremos seguidamente. A modo de clave, y como guía en la interpretación, cada categoría se interrelacionó con otra o con sus subcódigos de manera directa e indirecta. Las relaciones directas se interpretan con una línea recta y aquellas indirectas (coocurrencias) con una línea discontinua. El grosor de cada línea relacional indica mayor presencia discursiva, por tanto, mayor fuerza semántica en la relación intercategoría.

\subsection{LA ESTIGMATIZACIÓN DE LA INFANCIA MIGRANTE DESDE LA DIFERENCIA}

En esta categoría, es posible interpretar diversas prácticas y discursos que generan una percepción de las y los otros, en este caso de las y los estudiantes migrantes, como diferentes, estableciendo un grupo estigmatizado por su origen nacional. En este sentido, se adhieren una serie de características culturales a distintos grupos de migrantes según su origen nacional, siendo la estigmatización de estos grupos la mayor relación discursiva directa en la categoría. Es decir, las diferencias percibidas por las y los profesionales circulan en una tensión entre lo nacional y lo extranjero. Esto es coherente con los estudios que muestran que por lo general la concepción de la infancia migrante y su tránsito por los sistemas educativos está asociada a déficits que operan en lo académico y conductual (Cerón et al., 2017). Sin embargo, para el caso de la infancia migrante haitiana se puede advertir 
es el principal objeto de estigma y del asistencialismo de la escuela según se distingue en los discursos de las y los profesionales. En el escenario actual, configuran una alteridad por excelencia. Estas y estos 'otros' son objeto de asistencia debido a sus condiciones de pobreza, particularmente en acciones caritativas de recolección de recursos básicos como uniformes o útiles escolares, dejando de lado la pobreza que pudiesen experimentar otros migrantes o nacionales. Este es un tema relevante en los contextos migratorios, porque instala la idea de competencia por los recursos, de por sí escasos, entre grupos empobrecidos, lo que es más fuerte cuando son los nacionales quienes quedan postergados. Estas acciones suelen molestar incluso a algunos docentes pues comprenden que otras y otros niños también necesita de ayuda o derivación médica, sin embargo, la prioridad se establece con relación a la población haitiana:

En el caso de los niños haitianos que llegaron este año, que tienen hartas dificultades, ha habido harto apoyo [...] Algunos [profesores] están un poco choreaos [sic] (...) En especial, a los niños migrantes de acogerlos acá, de entregarles valores, también, de ayudarlos. Muchas veces se les ayuda, hay alumnos que vienen sin ropa, no sin ropa, pero que no tienen ropa del colegio, los papás no tienen ni siquiera para comprar. Entonces acá se les compra, no sé, desde camisa, pantalón, la corbata, se les entrega... hay un ropero escolar que va en ayuda de ellos, materiales, útiles escolares (Leticia, Profesora, Comuna de Recoleta, Región Metropolitana).

Otra relación potente es cómo la estigmatización de la población migrante se observa en el trato que las y los estudiantes reciben. Dicho trato tendría un carácter asimilacionista en cuanto a la visión de la inclusión en donde se enuncia un trato 'igualitario' que no pretende asumir la diferencia cultural, si no, más bien, pretende la adaptación de las y los estudiantes migrantes a las prácticas culturales nacionales:

Tratamos, nosotros, de que ellos vean que todos son iguales, tienen las mismas oportunidades educativas y, desde el punto de vista humano, se les trata exactamente igual, aunque antes ellos venían con esa especie de actitud autodefensiva y ellos planteaban "¿por qué a mí se me castiga y a los otros no?”; “¿por qué usted hace diferencia?" "Ahora no, vienen entendiendo que no" (Julio, Profesor, Comuna de Antofagasta, Región de Antofagasta).

Sin embargo, muchas veces ocurre que este trato igualitario finalmente termina ocultando la diversidad, por tanto, se trata de un discurso que es funcional a las ideas de asimilación, lo que viene desde una concepción inicial que vincula la diversidad que representan los y las migrantes a un obstáculo que debe ser superado (Ortiz, 2008; Poblete y Galaz, 2017).

Otra fuerte relación discursiva aparece en las coocurrencias de las categorías discriminación en la intersección género-origen nacional y sexualización del género. Esta relación es particularmente poderosa porque se encuentra al exterior de las coocurrencias, y transita en los discursos a través de la estigmatización de la infancia migrante, especialmente, como se observa en el mapa, de la estigmatización de la infancia colombiana. Esta relación la abordaremos en el análisis de la siguiente categoría.

Otra relación interesante entre un ellos y un nosotros surge en la relación entre los códigos adecuación curricular, trato y malestar docente. A pesar de que esta categoría 
aparece en contadas veces en el discurso de las y los profesionales, es importante declarar que las y los docentes reconocen al currículo como un objeto que ha de comprender la diferencia cultural, étnica e histórica y asumirla como un elemento a considerar en la política curricular nacional. En concreto, las evidencias indican que por lo general no se cuenta con mecanismos que favorezcan adaptaciones o flexibilizaciones curriculares, dado que incluso la propia normativa no contempla "lineamientos técnicos ni procedimientos que permiten flexibilizar los criterios de evaluación y promoción de los alumnos que no cumplen con los requisitos del marco curricular común" (Duk y Loren, 2010, p. 189). Las y los docentes valoran la diversidad que hoy comporta la escuela, pero requerirían de elementos de apoyo:

Nos falta más apoyo con respecto a cómo tratar y, de alguna manera, cómo utilizar algunas estrategias en la sala de clases, para trabajar con niños migrantes (Ester, Profesora, comuna de Antofagasta, Región de Antofagasta).

En resumen, podemos advertir que la categoría Diferencias y comparaciones demuestra los sesgos de las y los docentes respecto del abordaje de la diferencia, lo que se observa, por ejemplo, en la estigmatización de las infancias migrantes, especialmente en cuanto a la sexualización de las niñas afrocolombianas, lo que abordaremos en profundidad en el siguiente apartado. A continuación, mostramos el mapa semántico donde se sintetizan las relaciones y discursos de esta categoría de análisis.

Mapa semántico 1. Categoría analítica 'Diferencias y comparaciones'

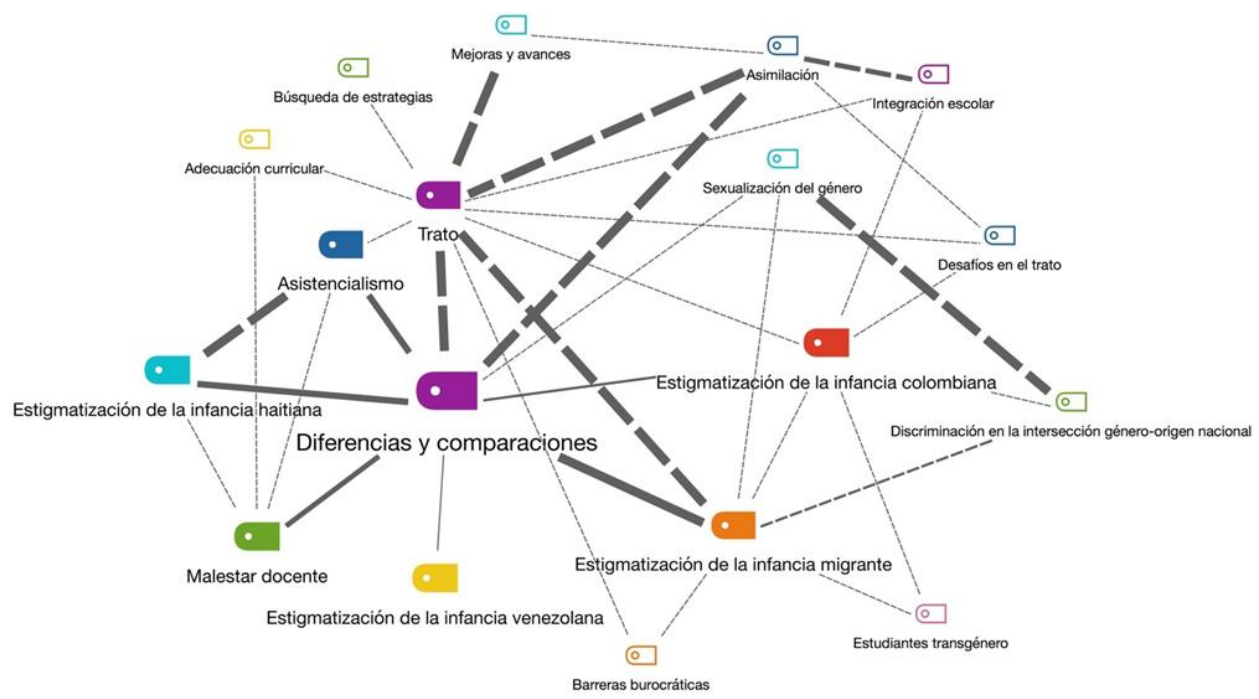

Fuente. Elaboración propia.

Como se observa, las relaciones directas de esta categoría ocurren en relación con los subcódigos asistencialismo, malestar docente, estigmatización de la infancia migrante, estigmatización de la infancia haitiana, estigmatización de la infancia venezolana, y 
estigmatización de la infancia colombiana. Las relaciones indirectas o de coocurrencia de la categoría se dan con los códigos trato y asimilación y cuya aparición de los discursos es fuerte. En cuanto a las relaciones semánticas directas más potentes nos encontramos con los códigos estigmatización de la infancia migrante, asistencialismo, y estigmatización de la infancia haitiana.

\subsection{LA INCLUSIÓN ESCOLAR Y LOS IMAGINARIOS RACIALES Y SEXUALES AL INTERIOR DE LA} ESCUELA.

Los discursos en esta categoría de análisis se orientan principalmente a dos aspectos relacionados con la inclusión escolar. El primero guarda relación con un trato y convivencia escolar que integra al migrante desde una mirada asimilacionista, y en el segundo aspecto se observan procesos de racialización y sexualización hacia la infancia migrante, particularmente sobre las niñas migrantes, estigmatizándolas. En cuanto al primer nodo crítico que nos presenta la categoría, podemos observar que los discursos de las y los profesionales de la educación dejan de manifiesto un abierto diálogo asimilacionista, en donde se entiende al trato que se da a las y los estudiantes nacionales como óptimo en comparación al que se podría atribuir. En este sentido, el buen trato se comprende desde una condición de poder, en donde el diálogo y la convivencia escolar no se encuentra en igualdad de condiciones, pues 'lo chileno' marcaría la puesta en marcha de condiciones dialógicas. Las y los profesionales igualan a través de ciertos marcadores discursivos como 'lo verdadero' a aquello que merece un trato adecuado, por tanto, el trato que las y los estudiantes migrantes podrían aspirar es mérito de la o el profesional al igualar su trato a las condiciones que merecen un buen trato, en este caso, lo chileno:

Son tratados como verdaderos niños chilenos (...) Así que son niños, como chilenos (...) Los menores de edad son más incluidos en nuestra cultura, que los adultos migrantes (Gabriel, Profesor, Comuna de Antofagasta, Región de Antofagasta).

En consecuencia, las y los profesionales comprenden que los derechos al buen trato y, por ende, todo lo que esto implica, atraviesa 'la chilenidad' o 'lo nacional', llegando a asumir que las condiciones de origen, identitarias y la condición de infancia pueden ser de algún modo rescatadas a través de la asimilación. Es decir, se es niño o niña si el comportamiento es similar al de las niñas y niños chilenos. Sobre este tema existen numerosos estudios que señalan que para las y los docentes es más fácil buscar la asimilación (Ortiz, 2008), dado que les resulta más cómodo trabajar con niñas y niños chilenos, ya que su formación inicial no los ha preparado para abordar el desafío de educar en la diversidad. Dentro de los procesos de asimilación que se observan en los discursos, las y los profesionales sostienen que la aculturación se facilita a menor edad. Este fenómeno se ha abordado extensamente en la literatura y coincide, por ejemplo, con los hallazgos de Gibson (1998), Takenoshita, Chitose, Ikegami y Ishikawa (2014) y Ronkainen, Vapalahti y Tessieri (2017), quienes, desde diversos contextos, concluyen que los procesos de aculturación generados por la escuela son más rápidos si comienzan a menor edad, por ejemplo, a través de la adquisición del idioma autóctono.

Por otra parte, pudimos observar que se adscriben una serie de características culturales a los grupos de estudiantes migrantes, tales como la violencia y el grito, pero que 
a medida que la escuela establece el trato asimilacionista, las y los estudiantes migrantes se 'mimetizan' con las y los estudiantes autóctonos.

Antes era mucho más notorio [la violencia], ahora se ha mimetizado un poco más (...) poco a poco, ellos van adquiriendo, van suavizando... (Julio, Profesor, Comuna de Antofagasta, Región de Antofagasta).

Pareciera entonces que la "chilenización” de niñas y niños migrantes es una condición para funcionar adecuadamente en la escuela, por lo que se deben hacer esfuerzos para lograrlo. Esto implica, por un lado, desconocer las culturas de origen de las y los estudiantes y, por otro, concebir la escuela como un dispositivo de poder (Foucault, 1977; Bernstein, 1990; Bourdieu y Passeron, 2001), que busca imponer un determinado código que se reconoce como legítimo, y para el cual se dispone de todos los medios. Un punto importante al interior de los discursos es el concepto de igualdad que, de acuerdo con lo dicho anteriormente, opera como igualdad en el código legítimo. Como ya mencionábamos anteriormente, no queda absolutamente claro hasta qué punto esta igualdad tiene que ver con la comprensión de la igualdad de derechos o de igualar (asimilar) las características del estudiantado migrante con el nacional:

Todos los alumnos son iguales (Paulina, Profesora, Comuna de Antofagasta, Región Antofagasta).

Es interesante ver cómo en los discursos opera el concepto de igualdad en tensión a la heterogeneidad, pues esta condición diversa se reconoce tanto desde la legislación, la política pública y desde el currículo. Evidentemente, las y los estudiantes son distintos y constituyen la riqueza de la diversidad en las aulas y en los centros escolares. Cada cual posee una identidad en la que confluyen diversas historias de vida, experiencias, bagajes culturales y religiosos, sexualidades, entre otras dimensiones de la identidad. Por tanto, esta aseveración rápida respecto de la igualdad de las y los estudiantes en contraposición a la diversidad que las y los profesionales reconocen, se funda, más bien, en una autoprotección discursiva en pos de la comprensión de la y el estudiante migrante como "diferente". Creemos que el solo reconocimiento de la diferencia es interpretado por las y los docentes como una carga negativa al tratarse de estudiantado migrante. Probablemente, el concepto les permite resguardar una imagen que si bien se reconoce como objeto de asistencialismo, imbrica también la imagen en igualdad de derechos, aunque este ejercicio opere más bien a nivel discursivo. En parte, puede comprenderse como una demostración del resguardo de la propia imagen en pos de la imagen atribuida a la persona migrante. Esta situación es común en los encuentros interculturales desde la óptica de la Face negotiation theory de Ting-Toomey (2005), en donde esta interacción y comprensión de la identidad activa una serie de mecanismos en pos de sostener, desafiar o apoyar la identidad de la otra o el otro (Ting-Toomey y Kurogi, 1998). Por tanto, los conceptos aquí indagados dan cuenta de este tipo de encuentros en el contexto chileno de contacto migrante.

Un segundo nudo crítico en la categoría guarda relación con la racialización, sexualización y estigmatización de las y los migrantes. Estos fenómenos ya se han observado en otros estudios (Riedemann y Stefoni, 2015; Pavez-Soto, 2016; Stefoni, Stang y Riedemann, 2016). En términos generales, y desde una interpretación interseccional, 
hemos identificado que las intersecciones de género y origen nacional son objeto de mayor discriminación, especialmente cuando se trata de niñas migrantes. En este sentido, se observan racismos de género atribuidos particularmente a las niñas colombianas. Diversos profesionales manifestaron una biologización del género, adscribiendo características sexualizadas y bioculturales a las niñas colombianas, lo que contribuye a su estigmatización.

Yo he escuchado comentarios, así como que hace una niña chilena respecto a... a una niña de ... sobre todo, como te digo, colombiana (Hugo, Profesor, Comuna de San Joaquín, Región Metropolitana).

Algunas que se tiñen, sí, harto, entonces, a veces, o también, como en su país hace mucho calor, ellas tienden a mostrar más piel (Ester, Profesora, comuna de Antofagasta, Región de Antofagasta).

En este caso, siguiendo a Foucault (1977), pudimos observar marcadores biopolíticos en los discursos, en donde el biopoder subvierte la imagen de las niñas migrantes a través de la sexualización y racialización de los comportamientos cotidianos y de la expresión de género (Pavez-Soto, 2016).

Bueno, a ver, las niñas son un poquito más complicadas para nosotros. Ellas traen costumbres que nosotros no estamos acostumbrados. Si se les dice: "pueden venir con falda", vienen con falda un poco exageradas en cuanto a que son muy cortitas las faldas, entonces ellas han tenido que aprender que tienen que ser más recatadas, cierto, porque si no, los profesores, los inspectores le van llamando mucha la atención, cierto, empezamos con ese juego, con ese problema y ahí se sienten muy discriminadas ellas. Además que la niña extranjera, especialmente la colombiana, uno la ve con uniforme y después la ve con ropa de calle y es una mujer, está mucho más desarrollada, una nenita de doce, trece, catorce años, ya es una mujer, prácticamente. Son muy desarrolladas. Entonces no saben, ellas no tienen consciencia de eso, parece y no tienen tampoco recato, no tienen recato. Les gusta mucho el baile, mover la cintura, la pelvis y todo eso, entonces eso es chocante cuando uno recién empieza como a ver estas cosas, pero es cosa que las niñas se vayan acostumbrando y eso va desapareciendo, en realidad, ha desaparecido bastante. Antes era mucho más notorio ese problema, acá (Carlos, Profesor, comuna de Antofagasta, Región de Antofagasta).

Como se observa en algunos enunciados ("muestran más piel", "se tiñen más el pelo", "son muy desarrolladas", "van llamando mucho la atención", "ya es una mujer" o "les gusta mucho mover la cintura") los discursos docentes crean una reconstrucción de ciertos cuerpos infantiles femeninos como un objeto sexual, que provoca y desafía las lógicas morales escolares, por lo tanto, debe ser disciplinado y asimilado a las supuestas costumbres recatadas de los cuerpos femeninos chilenos. En otros estudios (PavezSoto, 2016; Pavez, 2016) se ha comprobado que algunas niñas y jóvenes migrantes (con determinadas características, como cuerpos hiperracializados) son reconstruidas como sujetos hipersexualizados en un contexto donde las jerarquías de poder se interseccionan con las jerarquías de edad, origen étnico-nacional, fenotipo y clase social. En ese entramado 
de poder, no es casual que en la escuela las niñas migrantes afroamericanas también sean situadas como sujetos hipersexualizados:

Las niñas migrantes, a veces, traen su pelo, que es pelo afro y se ponen sus trencitas (Ester, Profesora, comuna de Antofagasta, Región de Antofagasta).

También esta carga de género, que, también, no es ajena que probablemente, por cultura, las chicas serán más, no sé, menos, más osadas, quizás, en términos de cómo enfrenta una relación de gustarse, de buscarse ¿ya? Eh, con los chiquillos y... como que eso, inmediatamente genera el tema de que ¡Ah! que son todas prostitutas (Hugo, Profesor, Comuna de San Joaquín, Región Metropolitana).

La racialización de las niñas migrantes se acompaña de la sexualización debido a su origen nacional y evidentemente, a su género. En ese sentido, opera una suerte de hipersexualización sobre las niñas, en especial afro descendientes, que se traduce en estigmas y, en algunas ocasiones en formas de acoso (Poblete y Galaz, 2017; Pavez-Soto, 2016).

Otro punto que nos parece interesante como un nuevo punto a investigar en el futuro, es el caso de las y los estudiantes migrantes transgénero. Pudimos atestiguar dos aspectos importantes: primero, el discurso de rescate y segundo, el homonacionalismo. Los discursos de rescate en el caso de las y los migrantes queer se refieren a aquellos discursos que se elaboran como 'salvataje' de una minoría que se encontraría en necesidad de protección, elementos que se ven potenciados aún más por una concepción homonacionalista de la sociedad de acogida, como abierta a lo queer, en contraposición a las sociedades homofóbicas de origen, contribuyendo, de este modo, al nacionalismo (Butler, 1993). El homonacionalismo y los discursos de rescate constituyen un campo relativamente novedoso de investigación sobre la movilidad transnacional, el que ha estado enfocado principalmente en las y los migrantes refugiados queer (Puar, 2007; Murray, 2014). Es revelador apreciar cómo estos conceptos ya aparecen en los discursos de docentes chilenos, lo que contribuye directa o indirectamente, en el caso nacional, a la racialización de la población migrante queer, más aún respecto de niñas y niños transgénero, como se observa en el siguiente relato:

Yo creo que hay culturas... por ejemplo, tenemos un niño que es transgénito (sic), que es claramente ¡transgénico! (sic) Y que... su cultura, que es colombiana, es súper intransigente... acepte ese tema. Y hemos tenido la discusión que, inclusive, que vecinos del mismo niño nos han ido a reclamar a la escuela, que nosotros estamos (...) aceptando, digamos, ciertas cosas, por ejemplo, que el niño, no sé, venga con la cartera y cosas, así. Para ellos es imperdonable eso (María Elena, Educadora Diferencial, Comuna de Antofagasta, Región Antofagasta).

En este apartado, podemos notar como el 'nosotras y nosotros', que en este caso es representado por la educadora diferencial de la escuela, se desmarca de una posición 'intransigente', en donde claramente los trazos de homofobia y transfobia son visibles como indicios de una cultura menor, la de 'ellas y ellos', la que es incapaz de aceptar la transexualidad. En este sentido, la escuela, como aparato social, contribuye a 'la liberación' 
de las y niñas y los niños migrantes transexuales, en cuyas familias de origen transnacional no pueden ser ellas y ellos mismos, permitiendo la libertad de la expresión de género al interior de su espacio. Es decir, la escuela en tanto espacio ubicado en el contexto nacional representa una supuesta apertura de la sociedad chilena a la transexualidad y la expresión de género, en general. Claramente, y como se ha discutido a través de la evidencia internacional, la liberación de países homofóbicos de parte de las y los migrantes queer transita por la asimilación a un contexto nacional supuestamente abierto -y blanco en el caso europeo y estadounidense-, pero que invisibiliza los crímenes de odio y la discriminación de la comunidad LGBTIQ+ (Peltonen y Jungar, 2018). Ciertamente, estas situaciones de exclusión se habían encontrado en el Norte Global, específicamente en solicitantes de refugio por orientación sexual, principalmente en los países nórdicos (Murray, 2014; Akin, 2016; Peltonen y Jungar, 2018; Eggebø, Stubberud y Karlstrøm, 2018; Keskinen, 2018). A continuación, se presenta el mapa semántico de esta categoría.

Mapa semántico 2: Categoría analítica 'Inclusión escolar'

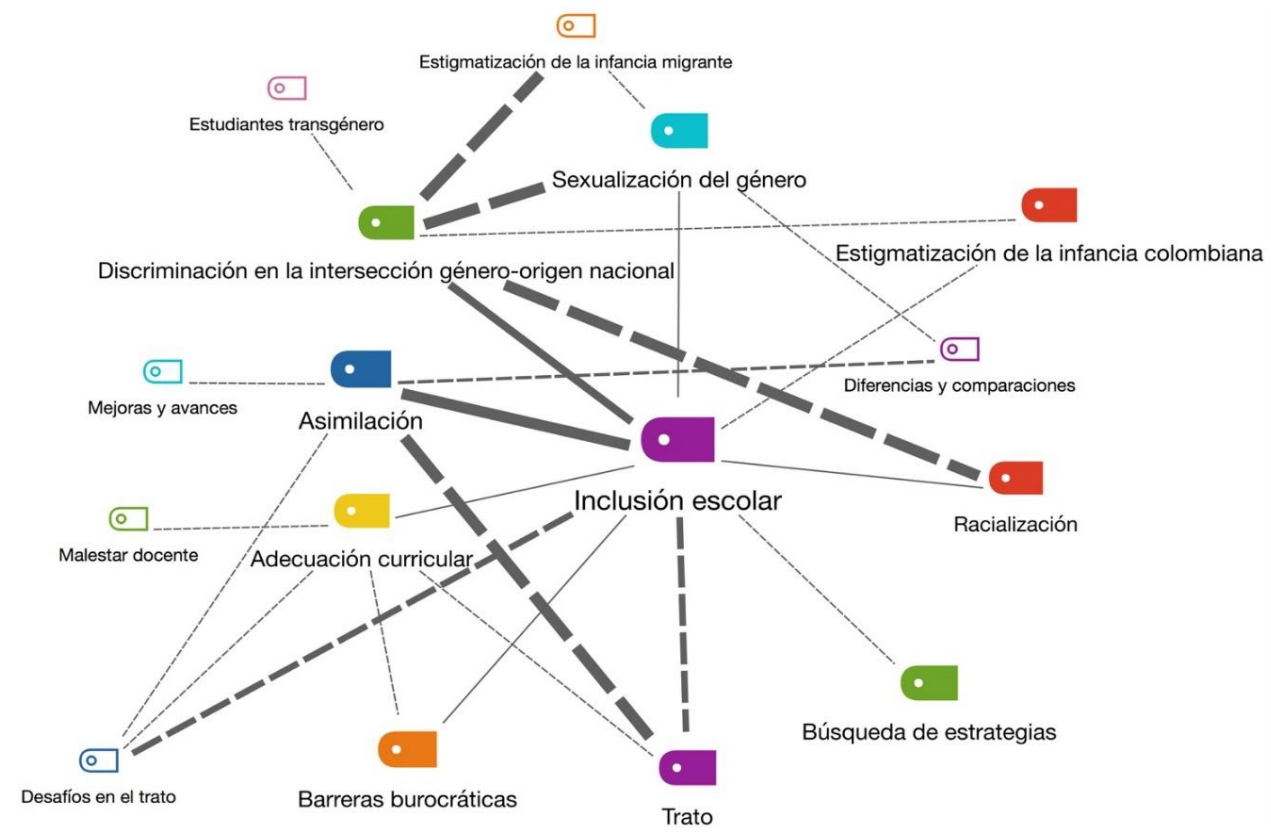

Fuente. Elaboración propia.

El mapa de la categoría Inclusión escolar está compuesto por los subcódigos asimilación, adecuación curricular, barreras burocráticas, discriminación en la intersección género-origen nacional, sexualización del género y racialización. Como relaciones indirectas o coocurrentes, la categoría se relaciona con los códigos desafios en el trato, trato y estigmatización de la infancia colombiana. Las relaciones semánticas más fuertes se observan en las relaciones con las categorías asimilación y discriminación 
en la intersección género-origen nacional en cuanto a relaciones directas. Respecto de estas mismas relaciones semánticas, éstas están poderosamente influidas por categorías coocurrentes como en el caso del trato hacia la categoría asimilación y en el caso de discriminación en la intersección género-origen nacional por las categorías racialización y sexualización del género en un primer nivel y, en un segundo, por la categoría estigmatización de la infancia migrante.

\subsection{EL TRATO COMO EJE DE LAS PRÁCTICAS SOCIALES ESCOLARES}

En esta categoría se puede advertir que las y los profesionales de la educación observan un cambio o mejora en el trato respecto a experiencias pasadas, porque valoran la apertura de la escuela hacia la diversidad, la que se nutre a través de la acogida de estudiantes migrantes. Sin embargo, esto no necesariamente implica un proceso dúctil:

Hemos desarrollado la tolerancia, hemos desarrollado la.... el acoger, en todo el sentido, al entender que ellos vienen de otro lado, sus formas, sus modismos (Julio, Profesor, Comuna de Antofagasta, Región de Antofagasta).

Por otro lado, el desarrollo de esta nueva habilidad docente y la apertura a la heterogeneidad de parte de la escuela ha permitido flexibilizar la estructura de la escuela como espacio social:

La apertura ha sido interesante, muy buena. Creo que ha sido súper positivo el trato que han tenido estos niños, porque les ha permitido, a los colegios, reformularse también (Pablo, Profesor, Comuna de Villa Alemana, Región Valparaíso).

Esta reformulación implica una búsqueda de nuevas estrategias en el trato, pues el imaginario de una escuela monocultural no se puede sostener en los contextos regionales en donde se encuentran ubicados los centros escolares en donde las y los profesionales se desempeñan:

Yo también he visto profesores súper comprometidos con alumnos extranjeros, bueno, en general, todos, se han hecho también charlas, hemos trabajado con [la ONG] Ciudadano Global, con otras entidades y otras redes de apoyo que nos ayudan, en este caso, a trabajar con los niños y darles un buen trato (Ester, Profesora, comuna de Antofagasta, Región de Antofagasta).

En los casos presentados aparece el valor de las actitudes positivas hacia la diversidad. De hecho, como se indicó antes, los distintos estudios llevados a cabo en contextos educativos concluyen en que las actitudes de las y los docentes hacia grupos minoritarios o marginalizados tienen una incidencia directa en las posibilidades de inclusión (Jordán, 1994; Poblete, 2009; León et al., 2007). Por otro lado, la exploración de redes de apoyo externas a la escuela ha sido crucial para el avance en el buen trato hacia las y los estudiantes migrantes. No obstante, esta búsqueda también entraña desencuentros y cavilaciones debido a la diversidad de estrategias y la posibilidad cierta de no encontrar una estrategia adecuada al contexto y a las necesidades estudiantiles: 
Muchos quieren, pero, como que también, el tema es que hay mil caminos más, entonces no llegan (Hugo, Profesor, Comuna de San Joaquín, Región Metropolitana).

Esta misma dificultad se ve reforzada a través de un escaso trabajo colaborativo o colegiado. Los docentes, en particular, manifiestan que los esfuerzos por encontrar las estrategias comunicativas y de convivencia adecuadas suelen ser individuales:

Eso [la atención al estudiantado migrante], en general, lo ve cada profesor jefe (Leticia, Profesora, Comuna de Recoleta, Región Metropolitana).

En consecuencia, la búsqueda de estrategias, aun cuando valorable desde el punto de vista pedagógico y ético, requiere comprenderse como un esfuerzo mancomunado, que demanda un fuerte liderazgo al interior de la escuela, el que permita que la búsqueda sea colectiva y apoyada institucionalmente. Este apoyo, además de local, debiese ser sistémico. Las y los docentes enuncian la necesidad de fortalecimiento de instancias de apoyo de parte de los organismos estatales:

Pero también quería agregar que de parte del Ministerio [de Educación] nos falta más apoyo (Ester, Profesora, comuna de Antofagasta, Región de Antofagasta).

En ese sentido, las evidencias muestran que las escuelas se sienten solas en el esfuerzo de educar y trabajar con estudiantes migrantes, por lo que terminan haciendo "lo que pueden" de manera intuitiva (Poblete y Galaz, 2017). La Política Nacional de Estudiantes Extranjeros 2018-2022 (Ministerio de Educación, 2018) no necesariamente ha redundado en acciones locales o en propuestas didácticas ministeriales oficiales, porque, en esa política se establecen una serie de objetivos en relación con la articulación institucional e intersectorial, el fortalecimiento de la gestión educativa y la vinculación territorial para el diseño de política pública. Sin embargo, la articulación pedagógica del currículo, por ejemplo, es omitida (Belmar et al., 2019). Por lo tanto, no es extraño que las y los docentes declaren la necesidad de una operacionalización concreta de recursos metodológicos orientados a la enseñanza-aprendizaje en contextos de acogida migrante, que emane desde el Ministerio de Educación en tanto organismo articulador de la política pública educacional, y que esté dirigida a cubrir las necesidades didácticas y de capacitación de las y los docentes. Por otro lado, las barreras burocráticas representan una dificultad en la inclusión de las y los estudiantes migrantes, siendo comprendida esta última como un desafío en el trato. En este sentido, existirían una serie de acciones que redundan en un trato diferente debido a su estatus migratorio:

También hay alumnos que, por problemas, digamos, burocráticos, no están en el listado de la JUNAEB, que tiene que ir la leche, al almuerzo, no están, porque tienen que pasar, primero, tienen que regularizar su situación y después ir a la municipalidad que les den el papel, el que acredite que ellos están en una situación miserable y, aquí, con ese papel se le da o tiene cupo para la alimentación. Pero, en muchos casos, los niños no tienen eso, porque recién están ingresando y, por ejemplo, en su casa no comen o comen súper poco. Entonces, acá lo que se hace es que, de alguna u otra manera, estirar las colaciones y, a veces, los niños que faltan, se ingresan estos alumnos extranjeros 
para dar su ración de desayuno, de almuerzo, algunas colaciones, se les dan colaciones, para que ellos no pasen por estas situaciones (Ester, Profesora, comuna de Antofagasta, Región de Antofagasta).

Los esfuerzos estatales llevados a cabo en el último tiempo como, por ejemplo, el $\mathrm{IPE}^{1}$, no implican la desaparición de los problemas que acarrea la condición irregular de las y los estudiantes migrantes en las situaciones escolares cotidianas. En este sentido, la escuela y sus profesionales se ven tensionados a suplir las necesidades básicas de las niñas y los niños migrantes, pues constituye un derecho. Además, la evidencia empírica respecto de las condiciones de seguridad alimentaria señala que, a mayores niveles de inseguridad y deprivación en el hogar, menores son los resultados de aprendizaje de las y los estudiantes, especialmente a nivel preescolar (Winicki y Jemison, 2003) y en países en vías de desarrollo (Aurino, Fledderjohann y Vellakkal, 2018). En consecuencia, mientras estas necesidades básicas no sean cubiertas de la mejor manera posible, lo que implica el compromiso estatal y político respecto de estas condiciones, es complejo avizorar y garantizar las condiciones mínimas para el aprendizaje. A continuación, se presenta el mapa semántico de esta categoría.

\section{Mapa semántico 3: Categoría analítica 'Trato'}

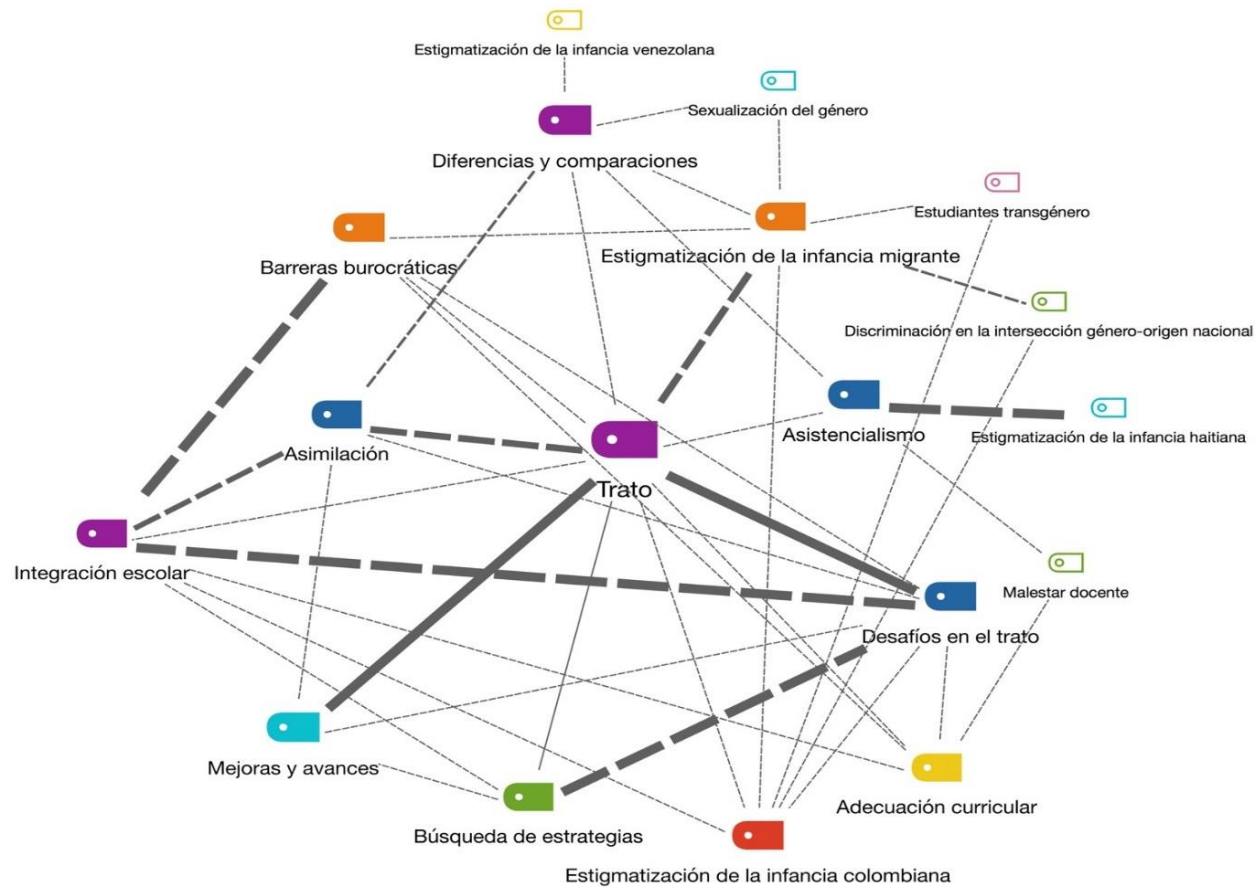

Fuente: Elaboración propia.

El Identificador Provisorio Educacional (IPE) es un número de registro dentro del sistema educativo, destinado para la población infantil migrante que se encuentra en situación administrativa irregular, de este modo se garantiza el acceso escolar mientras se gestiona la Cédula de Identidad (Ministerio de Educación y Centro de Estudios, 2018). 
Como se puede observar, este es el mapa más complejo de las tres categorías analíticas principales. Aunque aparentemente pudiese comprenderse el trato como un elemento comunicacional bastante liviano, su conceptualización y profundidad en el discurso docente son complejas. Esta complejidad se evidencia gráficamente en el entretejido con diversos subcódigos y códigos co-ocurrentes, los que confluyen en distintas direcciones, indicando que en el trato de las y los docentes y la escuela, en general, confluyen una serie de prácticas sociales y discursos que se despliegan en un proceso interactivo y comunicacional entre estudiantes migrantes y otros actores sociales en la escuela. Por tanto, esta dimensión presentó los mayores desafíos para el análisis.

De esta categoría emergieron tres subcódigos desde los textos: desafíos en el trato, mejoras y avances y búsqueda de estrategias. De estas relaciones directas, las más fuertes son mejoras y avances y desafíos en el trato, lo que evidencia que la búsqueda de estrategias para mejorar los procesos comunicativos con las niñas y los niños migrantes es débil y no aparece frecuentemente en los discursos de manera directa con la categoría. Respecto de las relaciones indirectas de esta categoría con códigos coocurrentes, las categorías de asimilación y estigmatización de la infancia migrante son las más potentes. Sin embargo, existen otras coocurrencias que se presentan más externamente a la categoría, pero que nutren de significado a las otras. Estas son las relaciones que se producen entre las categorías barreras burocráticas e inclusión escolar, inclusión escolar y desafíos en el trato, búsqueda de estrategias y desafíos en el trato, y finalmente, entre las categorías asistencialismo y estigmatización de la infancia haitiana.

\section{CONCLUSIONES}

A modo de cierre, quisiéramos señalar que la exploración de las categorías analíticas a través del análisis del discurso de las y los profesionales de la educación contiene elementos que nos permitieron distinguir sesgos, racismos y asistencialismos, así como también pudimos vislumbrar atisbos de un futuro prometedor en cuanto a la relación entre estudiantes migrantes y la escuela.

En primer lugar, respecto de los sesgos, un punto importante es la relación entre el asistencialismo y la estigmatización de la infancia migrante, en general. Como se examina en los análisis, las niñas y los niños migrantes son centro de paternalismos que, si bien es cierto, operan desde la visualización de sus necesidades, se les estigmatiza como objetos que reciben ayuda o caridad. Por tanto, la capacidad de agencia de las y los estudiantes migrantes para actuar sobre sus propias vivencias o dinámicas escolares y sociales está ausente en los discursos de las y los profesionales. Este mismo asistencialismo no parece ser bien visto por algunas y algunos docentes, quienes manifiestan su descontento al comparar las necesidades de niñas y niños migrantes con nacionales, las que serían abandonadas en pos de la prioridad sujeta al grupo migrante. Sin embargo, este descontento no guarda relación con la subyugación a través de la estigmatización de las y los estudiantes migrantes como receptores pasivos de ayuda, especialmente en cuanto a la infancia migrante haitiana, sino, más bien, se alinea con el asistencialismo dirigido a otros grupos migrantes o nacionales. Es decir, se observa que las niñas y los niños, en general, son comprendidos como sujetos pasivos y receptores.

Por otra parte, creemos importante destacar la aparición del racismo y la hipersexualización de ciertos cuerpos de niñas migrantes, especialmente en el caso de las 
niñas afrocolombianas. En este sentido, las niñas migrantes son subyugadas desde el discurso nacionalista, en donde la 'raza' aparece como concesión de la sexualidad. Este entrecruce o intersección de diversas jerarquías de poder manifiesta los mecanismos de adscripción y discriminación, en donde se articulan y reproducen estereotipos y prejuicios de las y los profesionales de la educación. En resumen, dentro de la estigmatización de la infancia migrante, las niñas migrantes se advierten como el grupo más marginalizado, mayormente las niñas afrocolombianas; lo que recrea situaciones estructurales de discriminación y exclusión sobre un grupo socialmente situado en diversos ejes de desigualdad.

Otro punto interesante es el levantamiento de discursos de rescate y el homonacionalismo en el caso de estudiantes migrantes transgénero. En este sentido, creemos que la apertura incipiente de la sociedad chilena hacia el reconocimiento de los derechos fundamentales de la comunidad LGBTIQ+ ha contribuido al surgimiento de nacionalismos y de nuevas racializaciones de las y los estudiantes migrantes queer, adscribiendo más mecanismos de discriminación sobre este grupo.

Asimismo, creemos que la formación inicial docente debe apuntar a la observación y creación de una escuela diversa, inclusiva e intercultural, pero también lo necesitan quienes ya ejercen. En este estudio, las y los docentes declaran abiertamente la necesidad de mayores redes de apoyo y capacitación por parte de los organismos estatales. Sin duda, esto implicaría, además, una reformulación de mallas curriculares.

Finalmente, así como aseguran los actores entrevistados, se perciben mejoras y avances tanto en el trato como en la capacidad de comprender la diversidad y la inclusión en la escuela. Ciertamente, el reconocimiento de la diversidad como un aspecto positivo contribuye a que la convivencia escolar y la comunicación cotidiana se facilite. Según las y los docentes, ha implicado desafíos en cuanto a la flexibilidad y adaptación a un contexto diverso, más han podido navegar dentro de esta multiplicidad de identidades de mejor manera a través del contacto en el tiempo. En este sentido, creemos que la exposición a la diversidad y su valoración dentro de los espacios escolares transcenderá en mejores prácticas docentes y de gestión, lo que redunde eventualmente en mejoras en la convivencia escolar y en los aprendizajes. Como se dijo anteriormente, se requiere de una propuesta didáctica ministerial que permita el desarrollo de estrategias de enseñanza-aprendizaje adecuadas a contextos de diversidad (Granada, Pomes y Sanhueza, 2013; Stefoni et al., 2016; Mondaca, Muñoz, Gajardo y Gairín, 2018), pues, a la fecha, las y los docentes ponen en juego la propia capacidad de adaptación profesional y de adecuación curricular, sin recibir entrenamiento formal. Por tanto, se requiere de medidas concretas en cuanto a capacitación docente y la puesta en marcha de protocolos metodológicos que faciliten el proceso de enseñanza-aprendizaje en contexto de acogida migrante.

\section{REFERENCIAS BIBLIOGRÁFICAS}

Agencia de Calidad de la Educación. (2017). Taller buen trato en el aula. Recuperado de http:// archivos.agenciaeducacion.cl/Taller_Buen_trato_en_el_aula_docentes.pdf. Fecha de consulta: 1 de marzo de 2019.

Akin, D. (2016). Queer asylum seekers: translating sexuality in Norway. Journal of Ethnic and Migration Studies, 43(3), 458-474. doi:10.1080/1369183x.2016.1243050.

Aurino, E., Fledderjohann, J., \& Vellakkal, S. (2018). Inequalities in Adolescent Learning: Does the 
Timing and Persistence of Food Insecurity at Home Matter? University Ca' Foscari of Venice, Dept. of Economics Research Paper Series No. 09/WP/2018. http://dx.doi.org/10.2139/ssrn.3155049

Belmar, M., Cornejo, J., Cornejo, C., Domínguez, J., Rioseco, M. \& Sanhueza, S. (2019). Diversidad en el aula: perspectiva de género y migración en el sistema educativo chileno. Espacios en Blanco. Revista de Educación, 29(1), 29-44.

Bernstein, B. (1990). Poder, educación y conciencia. Sociología de la transmisión cultural. Barcelona: El Roure.

Blanco, R. (2005). La equidad y la inclusión social: Uno de los desafíos de la educación y la escuela hoy. REICE. Revista Iberoamericana sobre Calidad, Eficacia y Cambio en Educación, 4(3), $1-15$.

Booth, T. \& Ainscow, M. (2000). Guía para la evaluación y mejora de la ecuación inclusiva. Index for inclusion. Madrid: Consorcio Universitario para la Educación Inclusiva.

Bourdieu, P. \& Passeron, J. C. (2001). La Reproducción, Elementos para una Teoría del Sistema de Enseñanza. Madrid: Popular.

Butler, J. (1993). Critically Queer//Bodies That Matter: on the Discursive limits of «Sex». New York \& London: Routledge, 223-242.

Cerón, L., Pérez, M. y Poblete, R. (2017). Percepciones Docentes en torno a la Presencia de Niños y Niñas Migrantes en Escuelas de Santiago: Retos y Desafíos para la Inclusión. Revista Latinoamericana de Educación Inclusiva, 11(2) 233-246.

Departamento de Extranjería y Migración. (2017). Reportes Migratorios, Población Migrante en Chile. Recuperado de http://www.extranjeria.gob.cl/noticias/2017/09/11/dem-publica-completoreporte-sobre-la-poblacion-migrante-en-chile/. Fecha de consulta: 1 de marzo de 2019.

Duk, C. y Loren, C. (2010). Flexibilización del currículum para atender la diversidad. Revista Latinoamericana de Inclusión Educativa, 4(1), 187-210.

Dunn, K. C. \& Neumann, I. B. (2016). Undertaking discourse analysis for social research. University of Michigan Press.

Echeita, G. (2008). Inclusión y exclusión educativa. Voz y quebranto. REICE. Revista Iberoamericana sobre Calidad, Eficacia y Cambio en Educación, 6(2), 9-18.

Eggeb $\varnothing$, H., Stubberud, E. \& Karlstrøm, H. (2018). Queer Migrants in Norway - Key findings from a research project about living conditions among LGBTI persons in Norway. doi: 10.13140/ RG.2.2.15208.93440.

Foucault, M. (1977). Historia de la sexualidad I: La voluntad de saber. Madrid, España: Siglo XXI.

Granada, M; Pomes, M.P. \& Sanhueza, S. (2013). Actitud de los profesores hacia la inclusión educativa. Pap. trab. - Cent. Estud. Interdiscip. Etnolingüíst. Antropol. Sociocult. [online]. 25, 51-59.

Gibson, M. A. (1998). Promoting academic success among immigrant students: Is acculturation the issue? Educational Policy, 12(6), 615-633.

Giroux, H. (1994). Jóvenes, Diferencia y Educación Posmoderna. En: Castell et al. (1994) Nuevas Perspectivas Críticas en Educación. Barcelona: Paidós, 97-128.

Instituto Nacional de Estadísticas (INE) - Departamento de Extranjería y Migración (DEM) (2019). Estimación de Personas Extranjeras Residentes en Chile 31 de Diciembre 2018. En línea: https:// www.ine.cl/docs/default-source/demográficas-y-vitales/estimaciones-personas-extranjeras/ sintesis-estimacion-de-personas-extranjeras-residentes-en-chile.pdf?sfvrsn=14. Fecha consulta: 15 de febrero de 2019.

Jiménez, F. \& Fardella, C. (2015). Diversidad y rol de la escuela. Discursos del profesorado: en contextos educativos multiculturales en clave migratoria, Revista Mexicana de Investigación Educativa, 20(65), 419-441.

Johnson, D. (2015). Formar ciudadanos interculturales en un mundo global: Algunas notas desde los estudios curriculares. Revista Diálogo Andino, (47), 7-14. https://doi.org/10.4067/S071926812015000200002. 
Estudios Pedagógicos XLV, № 3: 163-183, 2019

PERCEPCIONES DE LA COMUNIDAD EDUCATIVA SOBRE ESTUDIANTES MIGRANTES EN CHILE: TRATO,

DIFERENCIAS E INCLUSIÓN ESCOLAR

Jordán, J. (1994). La escuela multicultural: Un reto para el profesorado. Barcelona. Paidós.

Kaluf, M. (2009). Niños inmigrantes peruanos en la escuela chilena (Tesis de Magister). Santiago de Chile: Universidad de Chile.

Keskinen, S. (2018). Territorial stigmatization, gendered racism and young people's agency in a multi-ethnic school. In Shefer, T., Hearn, J., Ratele, K., \& Boonzaier, F. (Eds.). Engaging Youth in Activism, Research and Pedagogical Praxis: Transnational and Intersectional Perspectives on Gender, Sex, and Race. Routledge.

Krippendorff, K. (2018). Content analysis: An introduction to its methodology. Sage publications.

León, B., Mira, A. y Gómez T. (2007). Evaluación de las opiniones sobre la inmigración y la multiculturalidad en la escuela de alumnos de magisterio. Revista Electrónica de Investigación Psicoeducativa, 5(12), 259-282.

McCarthy, C. (1994). Racismo y currículo: La desigualdad social y las teorías y políticas de las diferencias en la investigación contemporánea sobre la enseñanza. Madrid: Morata.

Ministerio de Educación, Centro de Estudios. (2018). Mapa del estudiantado extranjero en el sistema escolar chileno (2015-2017). Documento de trabajo $\mathrm{N}^{\circ} 12$. Santiago: MINEDUC.

Ministerio de Educación. (2018). Política nacional de estudiantes extranjeros 2018-2022, Santiago: MINEDUC.

Ministerio de Educación. (2011). Encuesta nacional prevención, agresión y acoso escolar Simce 2011. Recuperado de: http://pazeduca.cl/wp-content/uploads/2017/01/SIMCE-2011-Encuestanacional-prevenci\%C3\%B3n-agresi\%C3\%B3n-y-acoso-escolar-Mineduc.pdf Fecha de consulta: 1 de marzo de 2019.

Mondaca, C., Muñoz, W., Gajardo, Y. \& Gairín, J. (2018). Estrategias y prácticas de inclusión de estudiantes migrantes en las escuelas de Arica y Parinacota, frontera norte de Chile. Revista de Estudios atacameños, (57), 181-201. https://dx.doi.org/10.4067/S0718-10432018005000101.

Monge, V. (2015). La codificación en el método de investigación de la grounded theory o teoría fundamentada. Innovaciones educativas, 17(2), 77-84.

Murray, D. A. (2014). The (not so) straight story: Queering migration narratives of sexual orientation and gendered identity refugee claimants. Sexualities, 17(4),451-471. doi:10.1177/1363460714524767.

Novaro, G. (2016). Migración boliviana, discursos civilizatorios y experiencias educativas en Argentina. Nómadas, (45), 105-121.

Ocampo, A. (2016). La educación inclusiva en el siglo XXI: un análisis sobre sus formas condicionales y consecuencias teóricas más relevantes. Investigación y formación pedagógica, Revista del CIEGC, 3, 7-34.

Ogbu, J. (1991). Immigrant and involuntary minorities in comparative perspective. En M. Gibson y J. Ogbu (Coords.), Minority status and schooling. A comparative study of immigrant and involuntary minorities (pp. 3-33). Nueva York, NY: Garland Publishing.

Ortiz, M. (2008). Inmigración en las aulas: Percepciones prejuiciosas de los docentes. Revista de Sociología Papers, 87, 253-268. https://doi.org/10.5565/rev/papers/v87n0.805.

Ortiz-López, J. E. (2017). El diálogo como apuesta ética en las escuelas pluralistas. Teoría de la Educación, 29(2), 25-39. http://dx.doi.org/10.14201/teoredu2922539.

Pavez, J. (2016). Racismo de clase y racismo de género: "mujer chilena", "mestizo blanquecino" y "negra colombiana" en la ideología nacional chilena. En M. E. Tijoux, Racismo en Chile. La piel como marca de la inmigración, 227-241.

Pavez-Soto, I. (2016). Violencia sexual contra niñas migrantes en Chile: polivictimización, género y derechos, Rumbos TS, XI(14), 113-131.

(2012). Racismo e inmigración en Chile. Experiencias de la niñez peruana en Santiago de Chile. Si somos americanos. Revista de Estudios Tranfronterizos, 21(1), 75-99.

Pavez-Soto, I., Ortiz-López, J.E., Olguín, C., Jara, P. \& Domaica, A. (2018). Infancia migrante haitiana: barreras y oportunidades en el proceso de escolarización. EntreDiversidades, (11), 7197. https://doi.org/10.31644/ED.11.2018.a03. 
Peltonen, S. \& Jungar, K. (2018). The ascendency of whiteness: on racialiazed queerness in LGBTIQ refugee work. En Shefer, T., Hearn, J., Ratele, K. \& Boonzaier, F. (Eds.). Engaging Youth in Activism, Research and Pedagogical Praxis: Transnational and Intersectional Perspectives on Gender, Sex, and Race. Routledge.

Peña-Sandoval, C. (2017). The Remix of Culturally relevant pedagogy: pertinence, possibilities, and adaptations for the chilean context. Perspectiva Educacional, 56(1), 109-126.

Poblete, R. (2018). El trabajo con la diversidad desde el currículo en escuelas con presencia de niños y niñas migrantes: estudio de casos en escuelas de Santiago de Chile. Perfiles Educativos, $X I(159), 51-65$.

Poblete, R. (2009). "Educación intercultural en la escuela de hoy: reformas y desafíos para su implementación", Revista Latinoamericana de Educación Inclusiva, 3(2), 181-200.

Poblete, R. y Galaz, C. (2017). Aperturas y cierres para la inclusión educativa de niños/as migrantes en Chile. Revista Estudios Pedagógicos, XLIII(3), 239-257.

Puar, J. K. (2007). Terrorist assemblages: Homonationalism in queer times. Duke University Press.

Riedemann, A. \& Stefoni, C. (2015). Sobre el racismo, su negación, y las consecuencias para una educación anti-racista en la enseñanza secundaria chilena. Polis, Revista Latinoamericana, (42).

Ronkainen, J., Vapalahti, K. \& Tessieri, E. (2017). 7 Exploring migration and multicultural education in Finland. The Shifting Global World of Youth and Education, 98-111.

Stake, R. (2010). Investigación con estudio de casos. Madrid: Morata.

Stefoni, C., Stang, F. \& Riedemann, A. (2016). Educación e interculturalidad en Chile: Un marco para el análisis. Estudios internacionales, 48(185), 153-182. https://dx.doi.org/10.5354/07193769.2016.44534.

Superintendencia de Educación. (2017). Discriminación en la Escuela, Descripción y análisis a partir de denuncias de discriminación recibidas en la Superintendencia de Educación, presentes en las escuelas de nuestro país. Recuperado de: https://oei.cl/uploads/files/news/publications/92/ INFORME_FINAL_DENUNCIAS-_DISCRIMINACION_SUPEREDUC.pdf. Consultado el 1 de marzo de 2019.

Ting-Toomey, S., \& Kurogi, A. (1998). Facework competence in intercultural conflict: An updated face-negotiation theory. International Journal of Intercultural Relations, 22(2), 187-225. doi:10.1016/s0147-1767(98)00004-2.

Ting-Toomey, S. (2005). The Matrix of Face: An Updated Face-Negotiation Theory. En W.B. Gudykunst (Ed.), Theorizing About Intercultural Communication (pp. 71-92). Thousand Oaks, CA: Sage.

Takenoshita, H., Chitose, Y., Ikegami, S. \& Ishikawa, E. A. (2014). Segmented assimilation, transnationalism, and educational attainment of Brazilian migrant children in Japan. International Migration, 52(2), 84-99.

UNICEF. (2004). Convivencia y discriminación en el ámbito escolar. Santiago: UNICEF.

UNICEF. (2011). Convivencia y discriminación en el ámbito escolar. Santiago: UNICEF.

Valles, M. (2003 [1997]). Técnicas cualitativas de investigación social: reflexión metodológica y práctica profesional. Madrid: Síntesis.

Vogt, W. P., Gardner, D. C. \& Haeffele, L. M. (2012). When to use what research design. Guilford Press.

Winicki, J., \& Jemison, K. (2003). Food insecurity and hunger in the kindergarten classroom: its effect on learning and growth. Contemporary economic policy, 21(2), 145-157. 
